Article

\title{
Evaluation of Sex-Specific Gene Expression in Archived Dried Blood Spots (DBS)
}

\author{
James H. Resau ${ }^{1, \dagger}$, Nhan T. Ho ${ }^{2}$, Karl Dykema ${ }^{3}$, Matthew S. Faber ${ }^{4}$, Julia V. Busik ${ }^{4}$, \\ Radoslav Z. Nickolov ${ }^{5}$, Kyle A. Furge ${ }^{3}$, Nigel Paneth ${ }^{2,6}$, Scott Jewell ${ }^{1}$ and Sok Kean Khoo ${ }^{7, \dagger, *}$
}

1 Program of Biospecimen Science, Van Andel Research Institute, Grand Rapids, MI 49503, USA; E-Mails: james.resau@vai.org (J.H.R.); scott.jewell@vai.org (S.J.)

2 Department of Epidemiology \& Biostatistics, College of Human Medicine, Michigan State University, East Lansing, MI 48823, USA; E-Mails: nho@epi.msu.edu (N.T.H.); paneth@epi.msu.edu (N.P.)

3 Laboratory of Computational Biology, Van Andel Research Institute, Grand Rapids, MI 49503, USA; E-Mails: karl.dykema@vai.org (K.D.); kyle.furge@vai.org (K.A.F.)

4 Department of Physiology, Michigan State University, East Lansing, MI 48824, USA; E-Mails: fabermat71686@gmail.com (M.S.F.); busik@msu.edu (J.V.B.)

5 Department of Mathematics \& Computer Science, Fayetteville State University, Fayetteville, NC 28301, USA; E-Mail: rnickolov@uncfsu.edu

6 Department of Pediatrics \& Human Development, College of Human Medicine, Michigan State University, East Lansing, MI 48823, USA

7 Laboratory of Microarray Technology, Van Andel Research Institute, Grand Rapids, MI 49503, USA

$\dagger$ These authors contributed equally to this work.

* Author to whom correspondence should be addressed; E-Mail: sok-kean.khoo@vai.org; Tel.: +1-616-234-5536; Fax: +1-616-234-5537.

Received: 28 April 2012; in revised form: 25 July 2012 / Accepted: 30 July 2012 /

Published: 2 August 2012

Abstract: Screening newborns for treatable serious conditions is mandated in all US states and many other countries. After screening, Guthrie cards with residual blood (whole spots or portions of spots) are typically stored at ambient temperature in many facilities. The potential of archived dried blood spots (DBS) for at-birth molecular studies in epidemiological and clinical research is substantial. However, it is also challenging as analytes from DBS may be degraded due to preparation and storage conditions. We previously reported an improved assay for obtaining global RNA gene expression from 
blood spots. Here, we evaluated sex-specific gene expression and its preservation in DBS using oligonucleotide microarray technology. We found $\mathrm{X}$ inactivation-specific transcript (XIST), lysine-specific demethylase 5D (KDM5D) (also known as selected cDNA on Y, homolog of mouse (SMCY)), uncharacterized LOC729444 (LOC729444), and testis-specific transcript, Y-linked 21 (TTTY21) to be differentially-expressed by sex of the newborn. Our finding that trait-specific RNA gene expression is preserved in unfrozen DBS, demonstrates the technical feasibility of performing molecular genetic profiling using such samples. With millions of DBS potentially available for research, we see new opportunities in using newborn molecular gene expression to better understand molecular pathogenesis of perinatal diseases.

Keywords: archived dried blood spots (DBS); sex-specific; gene expression; molecular genetic profiling; microarray

\section{Introduction}

Newborn screening is a mandated and routine health care program in many countries such as the United States, Japan, and most European countries [1]. Blood is collected from a heel prick 24-48 h after birth, placed on a Guthrie card and air-dried before submission to laboratories for testing. Quantitative analytical assays for newborn screening range from simple approaches such as colorimetric assays to advanced technologies such as liquid chromatography-tandem mass spectrometry [2]. After newborn screening, unused blood spots and/or portions of blood spots remain, and these are either discarded or archived, depending upon prevailing law and practice. Archived dried blood spots (DBS) are kept under varied storage conditions (e.g., frozen or unfrozen) and for varied durations of time [1]. Some DBS have been kept since the beginning of newborn screening programs 30 years ago, and collections of DBS now number several millions in some US states. DBS represent a unique retrospective research material that comprehensively yet inexpensively captures virtually $100 \%$ of the newborn population, making it an ideal resource for molecular epidemiological studies.

The use of DNA and RNA as genomic templates for advanced molecular applications usually involves high-quality, non-degraded isolates from fresh and "unfixed" samples. However, when clinical material is the source, investigators must contend with imperfections in the sample stemming from fixation in surgical pathology paraffin blocks or use of other preservation methods, uncertain collection procedures, and in the case of many DBS samples, long-term storage at ambient temperatures. To maximize the public health value of the advanced molecular tools now available to examine such clinical material, it is important to develop, establish, and validate new molecular assays on such samples, and not only with ideal samples generated in well-controlled laboratory experiments. A particular challenge in relation to the use of DBS in molecular genomic studies is the presumed low quantity and degraded quality of nucleic acids that can be isolated from these samples. Nevertheless, DNA from DBS has been successfully isolated for molecular assays such as PCR, single nucleotide polymorphisms (SNPs) genotyping, genome-wide scanning and, direct sequencing [3-10]. RNA, however, is less stable under certain circumstances, degrades more easily than DNA, and can be more 
challenging to isolate from dried blood spots [11]. Nonetheless, several recent studies have detected RNA from viruses and naïve T-cells of dried blood spots using quantitative real-time PCR assays [12,13]. Our research team has also established molecular protocols for detecting global RNA gene expression from blood spot samples, both fresh and archived, using microarray technology [14,15].

Although improved assays have been established to attain global gene expression from DBS, the feasibility of acquiring differentially-expressed genes from one trait to another (e.g., diseased vs. healthy controls) to better understand their biological functions has yet to be proven. Here, we evaluate the fidelity of sex-specific RNA expression in DBS to show the feasibility of acquiring differentially-expressed genes from 75 males and 31 females.

\section{Results and Discussion}

\subsection{Differentially-Expressed Genes can Be Determined in DBS}

To address the concern of whether the overall RNA gene expression can be sufficiently preserved in DBS to answer significant biological questions, we investigated the gene expression of males vs. females acquired from DBS using microarray technology. We identified four statistically significant differentially-expressed genes between groups of male and female subjects. These genes were $\mathrm{X}$ inactivation-specific transcript (XIST), lysine-specific demethylase 5D (KDM5D) which is also known as selected cDNA on Y, homolog of mouse (SMCY), uncharacterized LOC729444 (LOC729444), and testis-specific transcript, Y-linked 21 (TTTY21) (Table 1). All genes were sex-related. The expression of XIST was lower in males than in females. KDM5D and LOC729444 were over-expressed in males compared to females while TTTY21 was under-expressed in females compared to males (Figure 1). Although other sex-related transcripts such as testis determining factor (TDF) could be detected, the gene expression was low in general and there was no significant difference between males and females.

Table 1. Four differentially-expressed genes that were statistically significantly different between males and females.

\begin{tabular}{cccc}
\hline Gene ID & $\begin{array}{c}\text { Average intensity in } \\
\left.\text { males (mean } \log _{2}\right)\end{array}$ & $\begin{array}{c}\text { Average intensity in } \\
\left.\text { females (mean } \log _{2}\right)\end{array}$ & Adjusted $\boldsymbol{p}$ value \\
\hline$X I S T$ & 5.73 & 6.92 & $1.6 \times 10^{-16 * * *}$ \\
KDM5D & 7.64 & 6.54 & $0.0036 * *$ \\
LOC729444 & 7.99 & 6.86 & $0.042 *$ \\
TTTY21 & 6.66 & 5.65 & $0.042 *$ \\
\hline
\end{tabular}


Figure 1. A heatmap showing four differentially-expressed genes between males $(n=75)$ and females $(n=31)$ which were statistically significant. Red indicates over-expressed transcripts, blue represents under-expressed transcripts, and grey represents absence of data due to unmet filtering criteria.

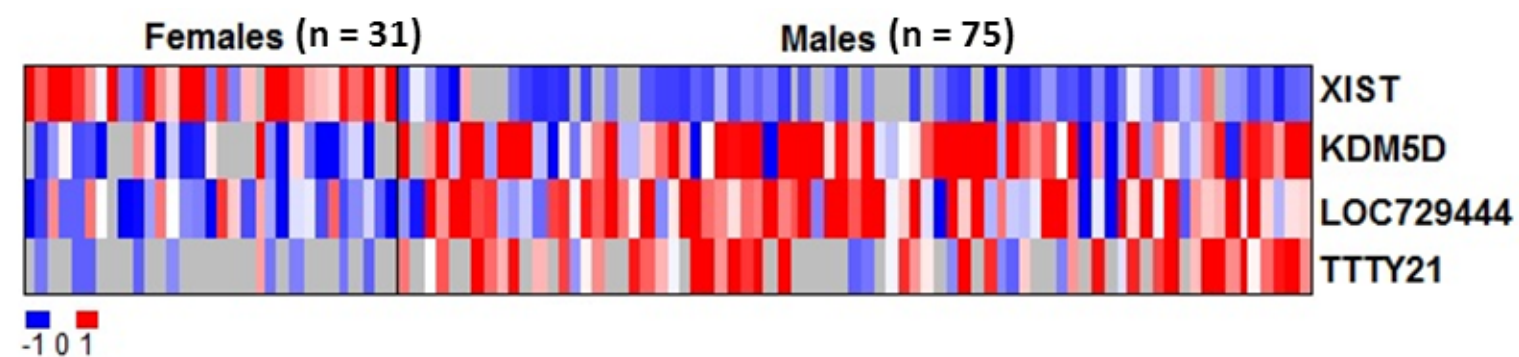

\subsection{RNAs Related to Sex Are Preserved in DBS}

Of the 4 genes that we identified, LOC729444 is an unknown gene while TTTY21 is a non-protein coding transcript. On the other hand, XIST and KDM5D are well-documented genes related to sex traits. Thus, we generated scatter plots using gene expression data (normalized probe intensities) of $X I S T$ and KDM5D to investigate whether RNAs related to sex is preserved. Our results demonstrated that the expression of XIST were generally higher in females than in males and vice versa for KDM5D. We confirmed that sexes can be distinguished and gender status is preserved in DBS (Figure 2A,B).

Figure 2. (A) Evidence of female-specific gene expression preservation in dried blood spots (DBS) by dot plot analysis of XIST. XIST gene probe intensities showed differentiation and preservation of its RNA gene expression in males vs. females; (B) Evidence of male-specific gene expression preservation in DBS by dot plot analysis of KDM5D. KDM5D gene probe intensities showed differentiation and preservation of its RNA gene expression in males vs. females.

A

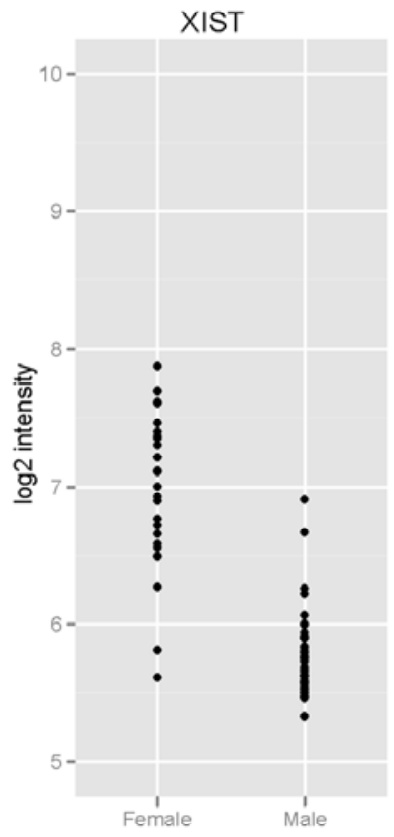

B

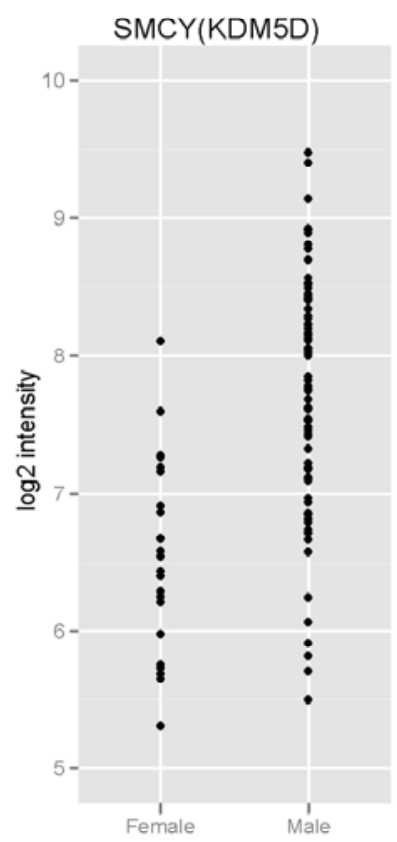




\subsection{XIST Expression can Be Validated Using qRT-PCR}

We validated the RNA gene expression of XIST, the most significantly differentially-expressed sex-specific gene, using qRT-PCR. In the microarray data, expression signal of XIST was weak in both sexes, but the expression signal in males was significantly lower than in females. The qRT-PCR result reflected this weak signal, in that we found a detectable XIST signal in only 12 of 31 females in our samples (38.7\%) (Figure 3A). However, detection of XIST by qRT-PCR was found only once in 75 male samples $(1.3 \%)$ (Figure $3 \mathrm{~B})\left(p<2.95 \times 10^{-6}\right.$; Fisher's exact test). Our observation confirmed the findings of microarray data that RNA gene expression of XIST is overall lower in males than in females.

Figure 3. qRT-PCR validation of XIST gene expression in (A) females and (B) males. $R n$ is the fluorescence reporter dye signal normalized to the fluorescence signal of ROX dye. $\Delta R n$ is $R n$ minus the baseline. Each curve represents the amplification linear plot of each sample.

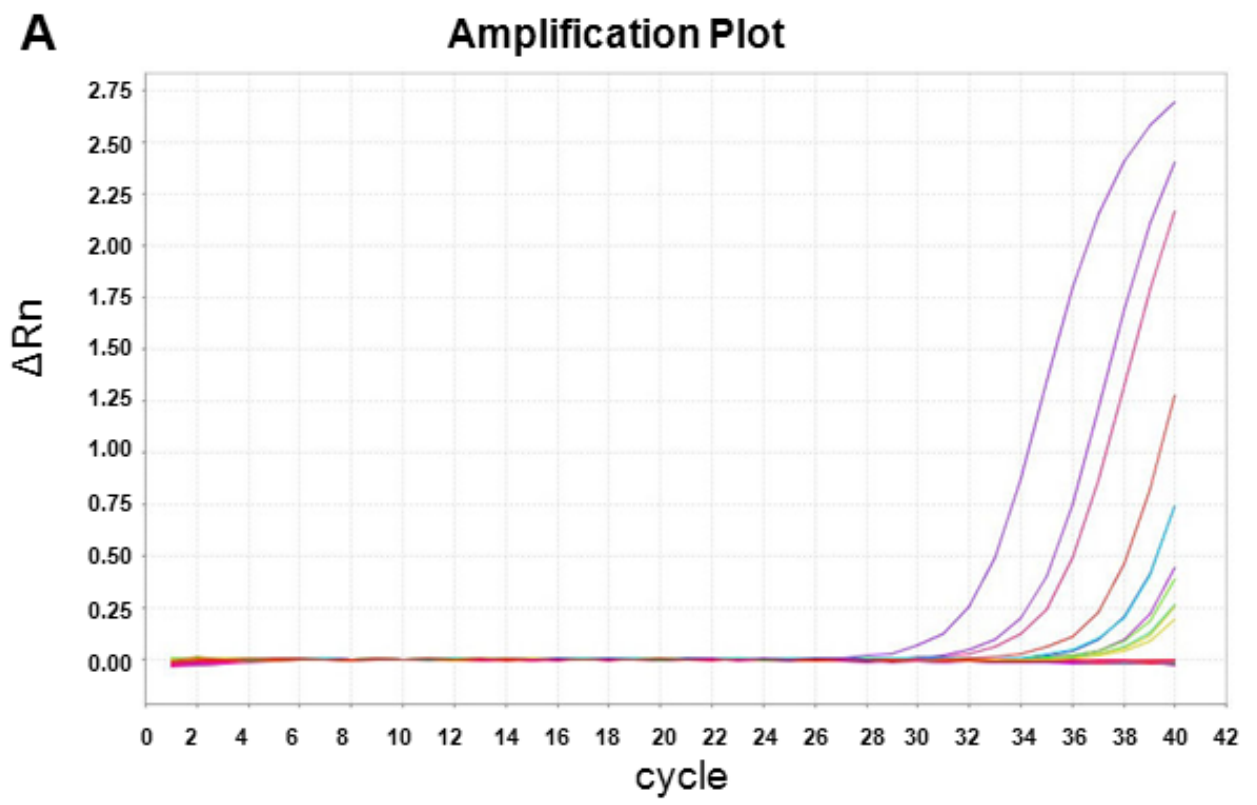

B Amplification Plot

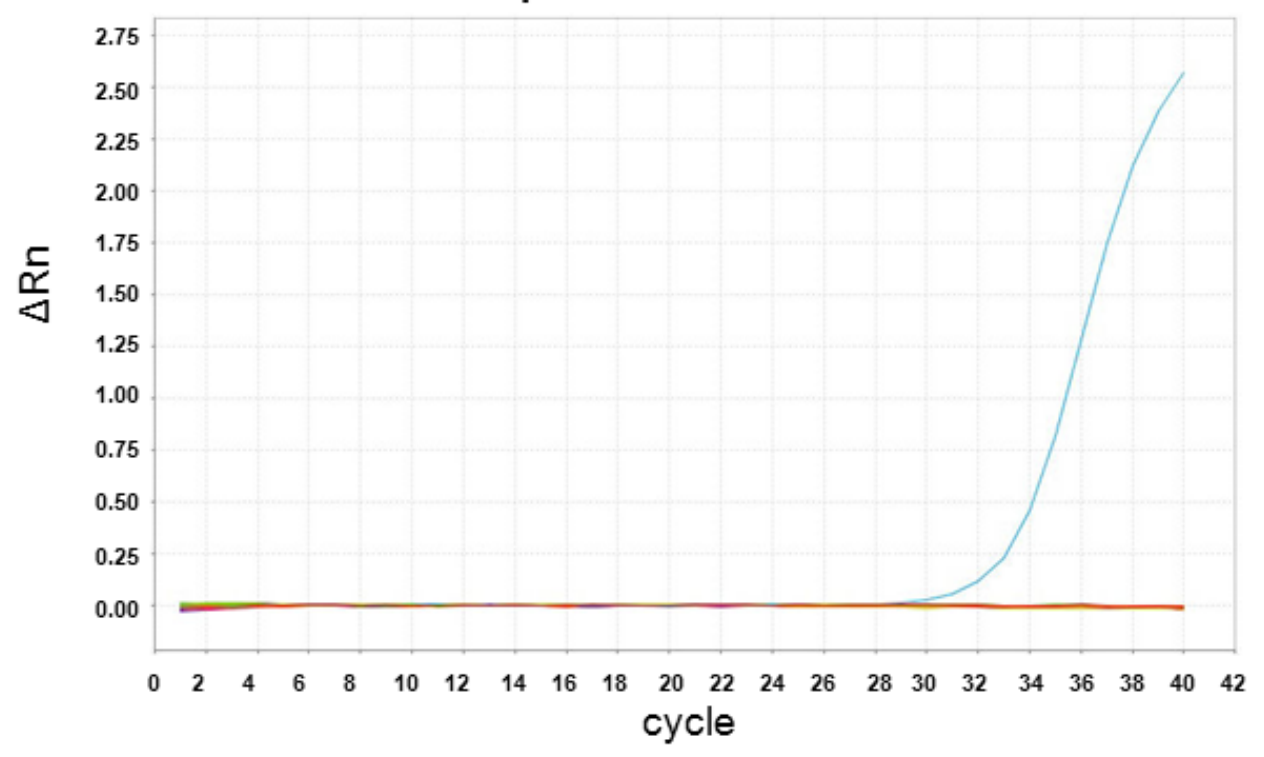




\subsection{Discussion}

It is a fact that high quality RNA is more difficult to acquire and store as it is more easily compromised when compared with DNA in human clinical samples. Therefore, it is logical to ask if any usable RNA can be obtained from DBS which are usually kept at ambient temperature. With the advancement of molecular tools and technologies, we recently established a technical protocol for using unique amplification systems to amplify non-poly A transcripts from compromised RNA and hybridizing them onto microarrays (with 60-mer length oligonucleotide probes) to obtain gene expression data from blood spot samples [15]. However, there remains the concern of whether the overall RNA gene expression can be sufficiently preserved in DBS to answer significant biological questions. To address this concern, we investigated the gene expression of 75 males and 31 females from DBS aged 2.5-15.6 years using microarray technology and evaluated their differentially-expressed genes. We identified 4 differentially-expressed genes which were statistically significant between males and females: XIST, KDM5D, LOC729444, and TTTY21. All four genes are sex-related.

$X I S T$, which is mapped on Xq13.2, is one of those genes involved in X chromosome inactivation [16]. The $\mathrm{X}$-inactivation center $(X I C)$ regulates $\mathrm{X}$-inactivation by counting the number of $\mathrm{X}$ chromosomes and contains XIST which localizes exclusively in the inactive X chromosome [17]. Thus, XIST is only expressed in the inactive chromosome $X$ of a female. Our results are in concordance with the above statement, showing significantly lower XIST expression $\left(p<1.6 \times 10^{-16}\right)$ in male than in female DBS samples. In addition, its gene expression from qRT-PCR was in concordance with the microarray data. KDM5D, also known as selected cDNA on Y, homolog of mouse (SMCY), is first isolated in the short arm of the mouse Y chromosome [18]. In mouse, its expression can be detected as early as in the 2-cell stage during blastocyst development. In the human, it is mapped on Yq11.222, encoding one human $\mathrm{H}-\mathrm{Y}$ (histocompatibility Y) epitope called H-Y/HLA-B7, and is proven to be Y-linked in many species [19]. In DBS, we also showed significantly higher KDM5D expression in our male samples as compared to our female samples $(p<0.0036)$. LOC729444 is an uncharacterized gene with currently unknown functions. It can be found in the cDNA libraries from testis according to the Cancer Genome Anatomy Project (http://cgap.nci.nih.gov/Genes). In our study, we also found LOC729444 highly expressed in males compared to females $(p<0.042)$. In addition, we found the expression of TTTY21, a non-protein coding RNA mapped on Yp11.2, significantly lower in females than in males $(p<0.042)$ from our DBS samples. We also examined the expression of these genes in fresh blood (male $n=102$, female $n=36$ ) and frozen tissues (male $n=39$, female $n=36$ ) that are available from the public domain. We and others found the expression of XIST significantly higher in females compared with males in fresh blood samples [20]. Generally, a higher intensity and fold-change are also observed in the fresh samples: 4 fold change in XIST $\left(p<4.7 \times 10^{-20}\right)$ and 3 fold change in KDM5D $\left(p<2.8 \times 10^{-23}\right)$, while LOC72944 and TTTY21 showed higher intensities but no significant fold-change $(p<0.022$ and $p<0.144$, respectively). Sex-specific genes including XIST and KDM5D were also reported to be differentially-expressed in fresh frozen male and female human brains [21]. Thus, although the overall intensity/fold-change is higher in fresh samples, gene expression changes of sex-specific transcripts in DBS are in concordance with those from fresh blood and frozen tissue samples in general. High-quality RNA extracted from matched whole blood at the time when DBS was collected could be used as a 
reference but unfortunately such samples are difficult, if not impossible to obtain, especially for very old DBS samples.

Overall, we showed that the expression of sex-related genes can be detected, measured, quantified, and correlated with their corresponding expression in each gender. The observed RNA gene expression and gender status preservation in DBS is confirmed by qRT-PCR. Preservation of gender status in DBS is both evidence of and an internal control for accurate gene expression related to certain traits that are preserved in DBS. Consequently, gene expression profiling can be performed to obtain genetic signatures to answer biological questions related to traits of interest.

\section{Experimental Section}

\subsection{Archived Dried Blood Spots (DBS)}

Archived dried blood spots (DBS) were obtained with parental consents from the Michigan Neonatal Biobank (MNB) which stores and manages Michigan's four million residual newborn dried blood spot cards (http://mnbb.org/). DBS from 53 pairs of cerebral palsy/healthy control subjects (75 male and 31 female) that had been archived from 2.5-15.6 years at ambient temperature were sampled for a large molecular epidemiological study of cerebral palsy using microarray technology. This study was approved by the Institutional Review Boards of Michigan State University, the Michigan Department of Community Health, and the Van Andel Research Institute.

\subsection{RNA and cDNA Preparation}

Total RNA was prepared according to our modified protocol [15]. In brief, three $3 \mathrm{~mm}$ punches from each blood spot were homogenized before total RNA extraction using the illustra RNAspin Mini RNA Isolation Kit (GE Healthcare, Buckinghamshire, UK). Total RNA was then concentrated with an RNA Clean \& Concentrator-5 Kit (Zymo Research, Orange, CA, USA). RNA quality and quantity were evaluated using an RNA Pico Lab Chip on the Agilent BioAnalyzer (Agilent Technologies, Santa Clara, CA, USA). The average RIN (RNA Integrity Number) was $2.3 \pm 0.71$. The WT-Ovation Pico RNA Amplification System (NuGEN Technologies, San Carlos, CA, USA) based on the Ribo-SPIA technology was used to amplify total RNA and synthesize first and second-stranded cDNA [22]. Unlike most standard amplification approaches which are based on T7 polymerase cRNA synthesis, this amplification system is initiated both at the $3^{\prime}$ end and randomly throughout the whole transcriptome. Hence, it has the advantage of amplifying non-poly A transcripts and compromised RNA from samples such as archived blood spots in our study.

\subsection{Labeling, Hybridization, and Scanning Procedures}

cDNA was labeled with Alexa Fluor 3 fluorescent dye from the BioPrime Total Genomic Labeling System (Invitrogen Life Technologies, Carlsbad, CA, USA) before purification using the PureLink PCR Purification System (Invitrogen). Purified labeled product was then applied onto a $8 \times 60 \mathrm{~K}$ whole human genome gene expression microarray (Agilent) for hybridization using standard Agilent procedures. After hybridization for $17 \mathrm{~h}$ at $65^{\circ} \mathrm{C}$ and $10 \mathrm{rpm}$ rotation speed, the arrays were washed for 2 min each with wash buffer 1 and 2 and scanned with an Agilent G3 high-resolution scanner. 
Probe features were extracted from the microarray scan data using Feature Extraction software v. 10.7.3.1 (Agilent).

\subsection{Gene Expression Microarray and Statistical Analyses}

All procedures for data processing and analysis were done in R version 2.13.2. Poor-quality signals on the Agilent microarrays were removed when the gProcessed signal was less than two times the gProcessed signal error [23]. Data were normalized between arrays using quantile normalization method [24-26]. Normalized data were then aggregated to gene level to assess differential expression of all individual genes between males and females by moderate $t$-statistic from linear model and empirical Bayes method implemented in the limma $\mathrm{R}$ package [25,26]. Significance of genes were adjusted for multiple testing using the Benjamini and Hochberg method [27]. Statistical significance was defined when adjusted two-sided $p \leq 0.05$. The median average of the expression values from the females $g(\mathrm{f})$ and males $g(\mathrm{~m})$ for each gene were computed to generate the heatmap. The group-wise average, $[g(\mathrm{f})+g(\mathrm{~m})] / 2$, was subtracted from each expression value to generate relative expression values for plotting.

\subsection{Quantitative Real-Time PCR Analysis ( $q R T-P C R)$}

To validate the microarray results, the most significantly differentially-expressed sex-specific gene, XIST, was evaluated using qRT-PCR using Hs01079824_m1 primer/probe set (Applied Biosystems, Carlsbad, CA, USA). In brief, 20 ng cDNA was mixed with Taqman PCR Super Mix (Applied Biosystems, Carlsbad, CA, USA) and gene-specific forward/reverse primers and probes before subjected to real-time PCR quantification using the ABI PRISM 7500 Fast Real-time PCR System (Applied Biosystems, Carlsbad, CA, USA). The data are presented as number of cycles to threshold.

\section{Conclusions}

In conclusion, we showed the evidence of RNA gene expression preservation and the technical feasibility of obtaining differentially-expressed genes in DBS. Since DBS represents a unique but underutilized research resource for molecular genomic studies, our study provides an important experimental technical advancement to facilitate exploration of DBS in molecular pathogenesis. The capability to obtain molecular genetic profiles (sets of differentially-expressed genes related to certain traits) from DBS offers the best possible baseline picture of a studied subject and can be a powerful molecular tool in allowing us to know possible disease etiology without the interference of post-natal factors such as environment or diet.

\section{Acknowledgments}

We thank all anonymous participants from Michigan for contributing their blood spot samples. We thank David Petillo for technical editing. This study is funded by the Van Andel Research Foundation and is also supported in part by NIH grant R01NS055101. 


\section{Conflict of Interest}

The authors declare no conflict of interest.

\section{References}

1. Green, N.S.; Pass, K.A. Neonatal screening by DNA microarray: Spots and chips. Nat. Rev. Genet. 2005, 6, 147-151.

2. Wilcken, B.; Wiley, V. Newborn screening. Pathology 2008, 40, 104-115.

3. Kammesheidt, A.; Kharrazi, M.; Graham, S.; Young, S.; Pearl, M.; Dunlop, C.; Keiles, S. Comprehensive genetic analysis of the cystic fibrosis transmembrane conductance regulator from dried blood specimens-Implications for newborn screening. Genet. Med. 2006, 8, 557-562.

4. Streetly, A.; Clarke, M.; Downing, M.; Farrar, L.; Foo, Y.; Hall, K.; Kemp, H.; Newbold, J.; Walsh, P.; Yates, J.; et al. Implementation of the newborn screening programme for sickle cell disease in England: Results for 2003-2005. J. Med. Screen. 2008, 15, 9-13.

5. Hollegaard, M.V.; Grove, J.; Thorsen, P.; Norgaard-Pedersen, B.; Hougaard, D.M., High-throughput genotyping on archived dried blood spot samples. Genet. Test. Mol. Biomark. 2009, 13, 173-179.

6. Hollegaard, M.V.; Thorsen, P.; Norgaard-Pedersen, B.; Hougaard, D.M. Genotyping whole-genome-amplified DNA from 3- to 25-year-old neonatal dried blood spot samples with reference to fresh genomic DNA. Electrophoresis 2009, 30, 2532-2535.

7. Hollegaard, M.V.; Grauholm, J.; Borglum, A.; Nyegaard, M.; Norgaard-Pedersen, B.; Orntoft, T.; Mortensen, P.B.; Wiuf, C.; Mors, O.; Didriksen, M.; et al. Genome-wide scans using archived neonatal dried blood spot samples. BMC Genomics 2009, 10, 297.

8. Hardin, J.; Finnell, R.H.; Wong, D.; Hogan, M.E.; Horovitz, J.; Shu, J.; Shaw, G.M. Whole genome microarray analysis, from neonatal blood cards. BMC Genet. 2009, 10, 38.

9. Ji, H.; Li, Y.; Graham, M.; Liang, B.B.; Pilon, R.; Tyson, S.; Peters, G.; Tyler, S.; Merks, H.; Bertagnolio, S.; et al. Next-generation sequencing of dried blood spot specimens: A novel approach to HIV drug-resistance surveillance. Antivir. Ther. 2011, 16, 871-878.

10. Winkel, B.G.; Hollegaard, M.V.; Olesen, M.S.; Svendsen, J.H.; Haunso, S.; Hougaard, D.M.; Tfelt-Hansen, J. Whole-genome amplified DNA from stored dried blood spots is reliable in high resolution melting curve and sequencing analysis. BMC Med. Genet. 2011, 12, 22.

11. Matsubara, Y.; Ikeda, H.; Endo, H.; Narisawa, K. Dried blood spot on filter paper as a source of mRNA. Nucleic Acids Res. 1992, 20, 1998.

12. De Crignis, E.; Re, M.C.; Cimatti, L.; Zecchi, L.; Gibellini, D. HIV-1 and HCV detection in dried blood spots by SYBR Green multiplex real-time RT-PCR. J. Virol. Methods 2010, 165, 51-56.

13. Lang, P.O.; Mitchell, W.A.; Govind, S.; Aspinall, R. Real time-PCR assay estimating the naive T-cell pool in whole blood and dried blood spot samples: pilot study in young adults. J. Immunol. Methods 2011, 369, 133-140.

14. Haak, P.T.; Busik, J.V.; Kort, E.J.; Tikhonenko, M.; Paneth, N.; Resau, J.H. Archived unfrozen neonatal blood spots are amenable to quantitative gene expression analysis. Neonatology 2009, 95, 210-216. 
15. Khoo, S.K.; Dykema, K.; Vadlapatla, N.M.; LaHaie, D.; Valle, S.; Satterthwaite, D.; Ramirez, S.A.; Carruthers, J.A.; Haak, P.T.; Resau, J.H. Acquiring genome-wide gene expression profiles in Guthrie card blood spots using microarrays. Pathol. Int. 2011, 61, 1-6.

16. Brockdorff, N.; Ashworth, A.; Kay, G.F.; Cooper, P.; Smith, S.; McCabe, V.M.; Norris, D.P.; Penny, G.D.; Patel, D.; Rastan, S. Conservation of position and exclusive expression of mouse Xist from the inactive X chromosome. Nature 1991, 351, 329-331.

17. Wutz, A.; Gribnau, J. X inactivation Xplained. Curr. Opin. Genet. Dev. 2007, 17, 387-393.

18. Agulnik, A.I.; Mitchell, M.J.; Lerner, J.L.; Woods, D.R.; Bishop, C.E. A mouse Y chromosome gene encoded by a region essential for spermatogenesis and expression of male-specific minor histocompatibility antigens. Hum. Mol. Genet. 1994, 3, 873-878.

19. Kent-First, M.G.; Maffitt, M.; Muallem, A.; Brisco, P.; Shultz, J.; Ekenberg, S.; Agulnik, A.I.; Agulnik, I.; Shramm, D.; Bavister, B.; et al. Gene sequence and evolutionary conservation of human SMCY. Nat. Genet. 1996, 14, 128-129.

20. Kim, S.J.; Dix, D.J.; Thompson, K.E.; Murrell, R.N.; Schmid, J.E.; Gallagher, J.E.; Rockett, J.C. Effects of storage, RNA extraction, genechip type, and donor sex on gene expression profiling of human whole blood. Clin. Chem. 2007, 53, 1038-1045.

21. Vawter, M.P.; Evans, S.; Choudary, P.; Tomita, H.; Meador-Woodruff, J.; Molnar, M.; Li, J.; Lopez, J.F.; Myers, R.; Cox, D.; et al. Gender-specific gene expression in post-mortem human brain: Localization to sex chromosomes. Neuropsychopharmacology 2004, 29, 373-384.

22. Watson, J.D.; Wang, S.; von Stetina, S.E.; Spencer, W.C.; Levy, S.; Dexheimer, P.J.; Kurn, N.; Heath, J.D.; Miller, D.M., III. Complementary RNA amplification methods enhance microarray identification of transcripts expressed in the C. elegans nervous system. BMC Genomics 2008, 9,84 .

23. Patterson, T.A.; Lobenhofer, E.K.; Fulmer-Smentek, S.B.; Collins, P.J.; Chu, T.M.; Bao, W.; Fang, H.; Kawasaki, E.S.; Hager, J.; Tikhonova, I.R.; et al. Performance comparison of one-color and two-color platforms within the MicroArray Quality Control (MAQC) project. Nat. Biotechnol. 2006, 24, 1140-1150.

24. Bolstad, B.M.; Irizarry, R.A.; Astrand, M.; Speed, T.P. A comparison of normalization methods for high density oligonucleotide array data based on variance and bias. Bioinformatics 2003, 19, 185-193.

25. Smyth, G.K. Linear models and empirical bayes methods for assessing differential expression in microarray experiments. Stat. Appl. Genet. Mol. Biol. 2004, 3, doi:10.2202/1544-6115.1027.

26. Smyth, G.K. Limma: Linear Models for Microarray Data. In Bioinformatics and Computational Biology Solutions using $R$ and Bioconductor; Gentleman, R., Carey, V., Dudoit, S., Irizarry, R., Huber, W., Eds.; Springer: New York, NY, USA, 2005; 397-420.

27. Benjamini, Y.; Hochberg, Y. Controlling the false discovery rate: A practical and powerful approach to multiple testing. J. R. Stat. Soc. B 1995, 57, 289-300.

(C) 2012 by the authors; licensee MDPI, Basel, Switzerland. This article is an open access article distributed under the terms and conditions of the Creative Commons Attribution license (http://creativecommons.org/licenses/by/3.0/). 